\title{
Desenvolvendo um modelo de gestão da diversidade étnico-racial para equipes de desenvolvimento de software
}

\author{
Michelle Borges Miranda ${ }^{1}$, Rafael Prikladnicki ${ }^{1}$ \\ ${ }^{1}$ Escola Politécnica - Programa de Pós-graduação em Ciência da Computação (PPGCC) \\ Pontifícia Universidade Católica do Rio Grande do Sul (PUCRS) \\ 90.619-900 - Porto Alegre - RS - Brazil \\ michelle.mirandadedu.pucrs.br, rafaelp@pucrs.br \\ Nível: Doutorado \\ Ano de ingresso no Programa: 2019 \\ Época prevista de conclusão: Dezembro/2022 \\ Qualificação: Fevereiro/2021 \\ WTDSoft
}

\begin{abstract}
Resumo. Diversidade nas equipes de desenvolvimento de software é um tema que vem ganhando atenção tanto de pesquisadores, quanto de organizações de Engenharia de Software. Pesquisas empíricas vêm demonstrando a importância de segmentos da diversidade, tal como gênero, para a comunidade da computação apresentando elementos que são inerentes à categoria gênero. De forma semelhante, o debate da diversidade étnico-racial na Engenharia de Software vem sendo colocado a fim de entender os desafios e beneficios que esta categoria de diversidade pode proporcionar para times de desenvolvimento de software. Pesquisas de gerenciamento da diversidade visam compreender esse mecanismo dentro de times e organizações e podem gerar como resultados modelos de gestão que visem gerenciar os conflitos e interesses dos grupos envolvidos. $O$ objetivo deste estudo é propor um modelo de gestão da diversidade para equipes de desenvolvimento de software com foco no grupo de diversidade étnico-racial, a partir de uma investigação sobre as implicações da diversidade étnico-racial na Engenharia de Software. Para alcançarmos esse objetivo, foi selecionado a pesquisa-ação como metodologia de pesquisa colaborativa entre academia e indústria, que será realizada em organizações pertencentes ao Parque Científico e Tecnológico da PUCRS (Tecnopuc), com a possibilidade de outros parques tecnológicos no Brasil. Como resultado deste estudo, pretendese propor um modelo de gestão de diversidade étnico-racial para equipes de desenvolvimento de software, compostos por um conjunto de práticas que contribuíram para a base de dados sobre pesquisa empírica na área da Engenharia de Software e como diretrizes para organizações que desejarem implementar este modelo de gestão em seus times.
\end{abstract}

Palavras-chaves. Diversidade, Inclusão, Engenharia de Software, Desenvolvimento, Representatividade 


\section{Caracterização do problema}

Diversidade e inclusão tornaram-se pautas recorrentes entre as organizações de Engenharia de Software (empresas, consultorias, eventos), tanto em relação ao aprofundamento da discussão sobre o tema, quanto na proposição de ações visando tornar os ambientes mais inclusivos e diversos. Isso porque, de acordo com [Bradley and Hebert 1997], equipes com diversidade são fundamentais e mais eficazes para resolução de problemas complexos, além de ampliar as possibilidades criativas devido às experiências de aprendizagem.

Entretanto, a inclusão da diversidade traz consigo não apenas benefícios mas também desafios para organizações, o que as tornam um tipo de ambiente propício para a gestão da diversidade. Trabalhos contemporâneos tratam a gestão da diversidade como uma gestão de competências, habilidades, gênero, experiência, idade, origem social ou cultural com o objetivo de melhorar a presença e o destino - em certos contextos profissionais - de pessoas pertencentes a determinados grupos sociais discriminados ou excluídos, visando também tornar a organização mais eficiente, aproveitando ao máximo essas diferenças [Chanlat et al. 2013].

A gestão da diversidade se faz necessária à medida em que cresce os interesses das empresas da área de Engenharia de Software em incluir diversidade nas equipes a fim de maximizar os benefícios promovidos por ela, fortalecer as políticas de justiça social da organização, e impactar diretamente as atividades de desenvolvimento de software.

Entre as dimensões que compõem a diversidade (tal como gênero, idade e cultura) a perspectiva étnico-racial é um dos aspectos conforme descreve [Page 2019]. De acordo com [Gomes et al. 2005], a diversidade ético-racial refere-se à população negra composta por pessoas pretas e pardas, que possui características inerentes a esse grupo, bem como os outros grupos de diversidade. Isso torna a discussão de diversidade e aspectos humanos na Engenharia de Software rica e complexa, pois a existência de singularidades permite observar em equipes de desenvolvimento de software os efeitos da inclusão desses grupos de diversidade.

Entretanto, as implicações da diversidade étnico-racial em times de desenvolvimento de software não foram exaustivamente exploradas, mesmo em países como o Brasil onde a proporção de pessoas negras na sociedade não reflete na área da tecnologia. Sendo assim, este trabalho tem como motivação o aprofundamento das discussões sobre gestão da diversidade étnico-racial e Engenharia de Software, a partir das seguintes questões de pesquisa: Quais as consequências visíveis da falta de diversidade étnico-racial em equipes de desenvolvimento de software?, Quais ações podem ser efetivadas a fim de contribuir com a inclusão de diversidade étnico-racial em equipes de desenvolvimento do software?. Desta forma, a partir de um esforço colaborativo entre academia e indústria através de uma pesquisa-ação, pretende-se propor um modelo de gestão da diversidade para equipes de desenvolvimento de software com foco no grupo de diversidade étnico-racial (principal objetivo deste estudo).

\section{Fundamentação teórica}

Desenvolvimento de software é um processo constituído pelo uso efetivo de linguagens de programação, ferramentas e um grande esforço coletivo e criativo para alcançar os objetivos desejados. Atividades de desenvolvimento de software dependem de pessoas 
que geralmente são organizadas em equipes onde as diversas habilidades interagem entre si de forma cooperativa e construtiva.

Em suas pesquisas, [Faraj and Sproull 2000] observaram que as equipes possuem a capacidade de responder a demandas complexas como um sistema completo em si, ainda que as tarefas estejam para além das capacidades individuais dos membros das equipes. Segundo esses autores, quando as equipes são o mecanismo principal para a realização de um trabalho, gerenciar a equipe é uma tarefa organizacional importante.

Entre os componentes observáveis sobre gestão de equipes, a composição é entendida como um dos elementos fundamentais para alcançar um desempenho satisfatório. Construir equipes altamente eficazes é uma estratégia que agrega valor ao negócio. Segundo [Bradley and Hebert 1997], um dos principais motivos que contribuem para o mau desempenho de projetos de software é a composição de equipe. Sendo assim, as equipes de desenvolvimento de software são bons exemplos relacionados a importância e aos desafios sobre gerenciamento de equipes, principalmente em decorrência dos custos empregados para construir equipes eficazes.

Estudos como os de [Bradley and Hebert 1997] investigam fatores necessários para construir equipes eficazes. Para eles, a não observância de fatores sobre aspectos humanos na composição de equipes pode impactar negativamente e diretamente seu desempenho. Em seus trabalhos, os autores exploram modelos, sugestões de práticas e fatores críticos a serem observados na composição de equipes, tais como: diversidade de personalidade, liderança, comunicação, coesão interna, e gerenciamento de expertise.

Entretanto, construir equipes altamente eficazes é uma tarefa complexa, sendo necessário combinar diversos aspectos e contornar suas particularidades. A diversidade é um desses aspectos. De acordo com [Bradley and Hebert 1997], as equipes devem ser diversas, possuidoras de uma variedade de conhecimentos, habilidades e perspectivas. Para os autores, complementando [Faraj and Sproull 2000], equipes com diversidade são fundamentais e mais eficazes para resolução de problemas complexos do que grupos mais homogêneos.

De acordo com [Page 2019] a diversidade pode ser examinada em dois grupos principais: cognitivo e identitário. Entretanto, debater apenas sobre diversidade no seu sentido geral pode resultar em um distanciamento ou mesmo apagamento dos diversos grupos que auxiliam a compor essa diversidade. Desta forma, identificar sobre quais grupos de diversidade estamos nos referindo e discutir sobre os aspectos que os caracterizam é fundamental para obter-se uma compreensão mais apurada sobre os dados que se levantam em relação ao grupo de diversidade em questão.

Dentro dos grupos de diversidade de identidade podemos inferir a diversidade étnico- racial. A diversidade étnico-racial segundo [Gomes et al. 2005] é a forma como alguns intelectuais referem-se ao segmento negro. De acordo com a autora o termo étnicoracial compreende a multiplicidade de dimensões e questões que envolvem a história, a cultura e a vida dos negros no Brasil, além das características físicas e a classificação racial. Essas questões das quais refere-se [Gomes et al. 2005] são as particularidades a serem observadas na composição de equipes eficazes e na gestão da diversidade conforme sugeriu [Bradley and Hebert 1997].

Para [Gomes et al. 2005] a expressão étnico-racial vem sendo adotada dentro dos 
contextos teórico e político tentando acabar com o impasse e dicotomia entre os conceitos de raça e etnia para se referir ao segmento negro. A autora acrescenta ainda que, para uma compreensão em profundidade das relações étnico-raciais deve-se considerar os processos identitários vividos pelos sujeitos, ou seja, o modo como esses se observam, identificamse e falam de si mesmos e do seu pertencimento étnico-racial.

Equipes diversas podem significar mais possibilidades criativas devido às experiências de aprendizagens, assim como podem revelar dificuldades relacionadas à maturidade da equipe diante de diferentes contextos. Trabalhar com diversidade requer a capacidade de atender diferentes necessidades, exigências, potenciais e desafios que a diversidade pode oferecer para uma equipe. Por esses motivos, algumas organizações de Engenharia de Software desenvolveram modelos para gerenciar a diversidade a fim de promover os benefícios e mitigar as complexidades que podem surgir dentro das equipes ([Aydemir and Dalpiaz 2018]; [Garrison et al. 2010]).

Segundo [Garrison et al. 2010], a utilização de modelos de gerenciamento da diversidade ajuda a explicar as percepções sobre as diferenças individuais e como estas diferenças podem afetar a confiança, desempenho e coesão da equipe de desenvolvimento de software. Sendo necessário, de acordo com [Hodgson et al. 2012], modelagens que explorem a diversidade, identifiquem seus benefícios e desafios e compreendam como ela afeta o desempenho das equipes, sugerindo ações que visem melhorar suas performances.

Embora perceba-se um número maior de estudos sobre diversidade na área de Engenharia de Software tal como os trabalhos de [Menezes and Prikladnicki 2018] e [Kohl and Prikladnicki 2018], a diversidade étnico-racial é pouco explorada em relação às suas nuances e contribuições para a área da computação envolvendo empresas e academia. Semelhantemente os trabalhos sobre gestão da diversidade de ([Aydemir and Dalpiaz 2018] e [Garrison et al. 2010]) não focam na diversidade étnicoracial no contexto de times de engenharia de software.

Trabalhos identificados sobre diversidade étnico-racial, tratam sobre o ensino da Engenharia de Software e estratégias de inclusão e retenção para grupos de diversidade étnico-racial nas universidades. Tais trabalhos sugerem currículo, métodos de ensino e critérios de avaliação, mesclando o conhecimento internacional, conhecimento e o contexto étnico local ([Fendler and Winschiers-Theophilus 2010], [Leonard et al. 2013]. O que possibilita um cenário cheio de desafios e oportunidades para academia e indústria diante do tema da diversidade étnico-racial.

\section{Metodologia}

De acordo com [Wohlin et al. 2003] na engenharia de software, os métodos empíricos permitem incorporar o comportamento humano à abordagem de pesquisa adotada e fornecem uma base científica importante, um dos principais paradigmas da abordagem empírica é a pesquisa qualitativa, que se preocupa com estudos no seu ambiente natural.

Este trabalho será conduzido através de uma parceria academia-indústria, observando e dialogando com equipes de desenvolvimento de software no seu ambiente de trabalho. Desta forma, foi escolhida como ferramenta de pesquisa uma metodologia qualitativa que permite que pesquisadores e objetos de estudo interajam juntos na construção da solução de um problema de pesquisa. 
Uma abordagem metodológica frequentemente utilizada para pesquisas que visam a parceria entre academia e indústria na solução de uma problemática real sentida pela empresa é a pesquisa-ação. Esta abordagem metodológica permite a participação da pesquisadora de forma colaborativa com os atores do objeto de estudo na identificação do problema, elaboração de diagnóstico e desenvolvimento de possíveis soluções ou melhorias para situações cotidianas ([Coughlan and Coghlan 2002]).

A pesquisa-ação possui uma abordagem clássica já familiar nas áreas e organizações de tecnologia para identificação de um problema, o planejamento e avaliação de ações. Entre os modelos de pesquisa-ação existentes, escolheu-se o modelo clássico proposto por [Susman and Evered 1978], que divide a pesquisa-ação em cinco estágios:

1. Diagnóstico: nesta etapa, os pesquisadores avaliam as situações atuais da organização, detectando problemas e identificando quais grupos de interesse estão disponíveis para refletir e elaborar as ações pretendidas. Este diagnóstico é executado em conjunto por pesquisadores e representantes da organização.

2. Planejamento de ações: nesta etapa, há uma grande ênfase na compreensão do problema, das proposições de ações e eventuais hipóteses da pesquisa.

3. Ação: momento em que os resultados são divulgados com as propostas sugeridas. A ênfase está na execução, com base nas discussões com os grupos.

4. Avaliação: nesta etapa, o objetivo é observar, avaliar e realizar as alterações necessárias. Pesquisadores e colaboradores envolvidos no processo revisitam toda a jornada, avaliam ofeedback e os resultados.

5. Aprendizagem:é a etapa de generalização dos aprendizados obtidos pelo estudo e análise dos resultados.

A pesquisa-ação pode ser conduzida através de múltiplos ciclos, ou seja, é possível ter diversas iterações durante o processo de pesquisa a fim de melhorar a solução desenvolvida. Dessa forma, ampliam-se as possibilidades de alcance dos objetivos a cada novo ciclo realizado, viabilizando o refinamento da solução proposta.

Baseada no trabalho de [Andersson and Runeson 2007] sobre estudos interativos e de [Susman and Evered 1978], esta pesquisa será conduzida por ciclos de processos em espiral evolutivo na pesquisa-ação. De acordo com a (Figura 1), cada ciclo é composto pelas cinco fases da pesquisa-ação, onde cada uma pode conter processos e ferramentas distintas. Durante um ciclo uma proposta é desenvolvida, implementada e avaliada. Essas avaliações são utilizadas para melhorar a solução desenvolvida iniciando um novo processo. O primeiro ciclo é entendido como uma atividade de modelagem seguido por outros ciclos caracterizados como exploratórios.

Serão consideradas diferentes funções para sujeitos divididos em grupos no processo de pesquisa conforme descrito na (Figura 1), onde a responsabilidade é mostrada em branco para os pesquisadores, cinza claro para a indústria e academia e cinza mais escuro para indústria. Sendo de responsabilidade dos pesquisadores coletar e analisar os dados, enquanto os profissionais são responsáveis pela ação e procedimentos de trabalho na organização.

\subsection{Universo e unidade de análise da pesquisa}

A unidade de análise desta pesquisa são os times compostos por sujeitos profissionais de tecnologia da informação (TI) que fazem parte de times de desenvolvimento de soft- 


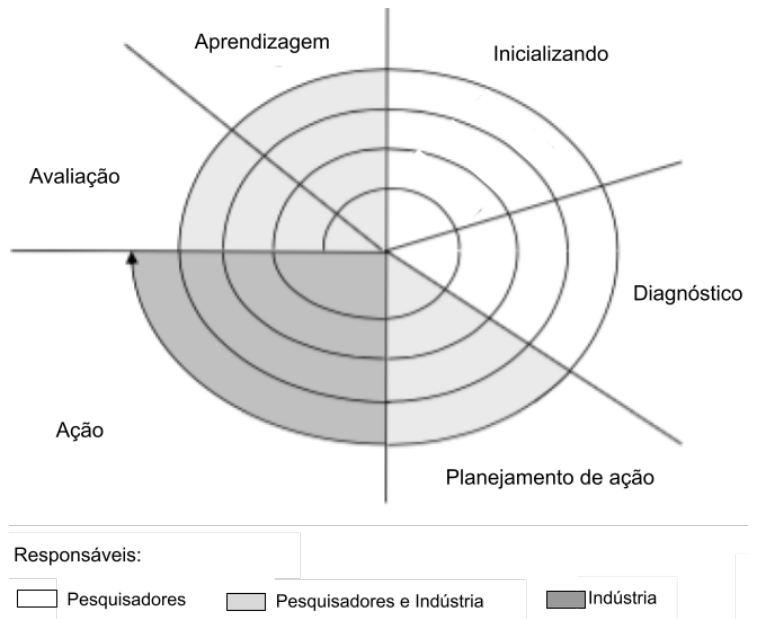

Figura 1. Modelo de ciclo em espiral adaptado de [Andersson and Runeson 2007] e [Susman and Evered 1978]

ware que possuam diversidade étnico- racial. Como trata-se de um estudo qualitativo, o tamanho da amostra será limitado.

Inicialmente planejamos coletar dados de empresas do Parque Científico e Tecnológico (Tecnopuc) pertencente à Pontifícia Universidade Católica do Rio Grande do Sul (PUCRS). Pretendemos realizar a pesquisa-ação em no máximo 10 empresas distintas do pertencentes ao Tecnopuc, sendo a unidade de análise a equipe de desenvolvimento de software, com a possibilidade de serem analisadas uma equipe de cada empresa.

\section{Método para avaliar resultados}

De acordo com [Susman and Evered 1978] a pesquisa-ação pode ser combinada com outras técnicas e metodologias de pesquisa para alcançar os objetivos propostos. Foram selecionadas algumas técnicas para auxiliar a coleta e análise de dados nas fases de diagnóstico, planejamento e avaliação conforme o modelo de pesquisa-ação utilizado.

Para a realização da etapa de diagnóstico e planejamento selecionamos como ferramenta de coleta e análise de dados o Lean Value Tree (LVT) de [J. Highsmith and Robinson 2019]. O LVT é uma técnica de investigação que auxilia os pesquisadores em: planejar e testar objetivos e ações; definir métricas, hipóteses e experimentos; gerenciar as opções de mudança e melhoria; compartilhar e analisar os resultados desejados, examinando riscos e oportunidades. O Lean Value Tree é dividido em quatro etapas (Figura 2):

- Definição da visão: Escolha do propósito do trabalho sistematizado em uma frase ou título de impacto.

- Objetivos: Definição dos objetivos a serem alcançados ao final da pesquisa.

- Apostas: Hipóteses formuladas que sugerem ações como resultados.

- Iniciativas: Conjunto de práticas elaboradas a serem desenvolvidas para alcançar as apostas sugeridas.

Dado que a pesquisa-ação é uma metodologia colaborativa, a ferramenta LVT será utilizada durante sessões de grupos com a participação e mediação dos pesquisadores. Essas sessões serão documentadas através de planilhas virtuais onde os sujeitos responderão 


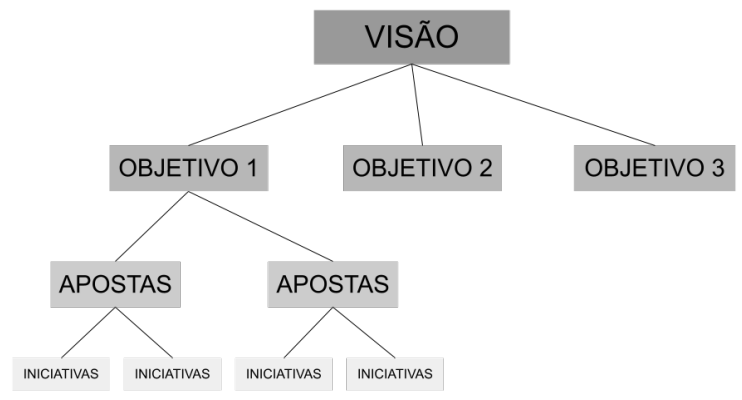

Figura 2. Etapas do LVT adaptado de [J. Highsmith and Robinson 2019]

as perguntas relacionadas ao LVT em planilhas virtuais em modo anônimo. Também serão utilizadas como ferramentas de coleta de análise de dados as notas de campo. Essas sessões serão conduzidas de forma que pesquisadores e participantes do estudo explorem, analisem e reflitam de maneira conjunta e colaborativa sobre o problema de pesquisa.

A sistematização dos resultados será realizada com o emprego do LVT que possibilita a geração do plano de ações. $O$ modelo de pesquisa-ação de [Susman and Evered 1978] é uma metodologia iterativa que prevê a avaliação dos resultados, dentro de um mesmo ciclo, realizada por pesquisadores e colaboradores do estudo.

Por avaliação de resultados na pesquisa-ação entende-se os feedbacks do plano de ação, necessários para melhoria da solução proposta. Os resultados numa pesquisa-ação podem ser observados pela proposta de solução do problema a ser investigado e/ou pela generalização dos dados (tipo de resultado característico pela academia).

Devido a este cenário, a avaliação dos resultados contará com o auxílio de três principais grupos necessários para a realização da pesquisa-ação conforme [Staron 2020]:

- Equipe ação: A equipe de ação é responsável pelo planejamento, execução e avaliação da pesquisa. Esse grupo é composto por representantes da empresa e pesquisadores.

- Grupo de referência: O grupo de referência é responsável pelo conselho e feedbacks para a equipe de ação.

- Equipe gestão: A equipe de gestão é responsável por gerenciar o projeto e a institucionalização das mudanças. Ambos grupos, de referência e de gestão, são compostos apenas por representantes da empresa.

\section{Estado atual do trabalho (resultados já existentes)}

Um primeiro ciclo foi realizado, servindo como base para os próximos ciclos exploratórios. Nessa primeira etapa foi realizado um estudo dentro de uma consultoria multinacional de tecnologia, localizada no Brasil. Essa primeira fase gerou um modelo inicial contendo métricas e ações para a gestão da diversidade étnico-racial em equipes de desenvolvimento de software.

O modelo de gestão da diversidade gerado foi categorizado em três processos: inclusão, desenvolvimento e representação. Este modelo foi desenvolvido a partir da colaboração entre pesquisadores. A íntegra da pesquisa-ação está publicada em:

- Michele Miranda, Rafael Prikladnicki. 2020. Towards a model for Managing Diversity and Inclusion in Software Development Teams. In 
34th Brazilian Symposium on Software Engineering (SBES '20), October 21-23, 2020, Natal, Brazil. ACM, New York, NY, USA, 7 pages. https://doi.org/10.1145/3422392.3422473

A partir dessa primeira experiência, pretende-se implementar o modelo gerado em outras empresas a fim de explorar o alcance dessas ações na promoção da inclusão de diversidade étnico-racial em times de desenvolvimento de software e com isso, evoluir o modelo de gestão a cada novo ciclo observando nuances tais como: contextos organizacionais; maturidade dos times sobre diversidade; entre outras diferenças entre as organizações que podem impactar na avaliação e generalização dos resultados da pesquisa.

\section{Contribuições esperadas}

Diante do exposto ao longo do trabalho, entende-se que a diversidade adquiriu um valor de negócio para diversas empresas de tecnologia, além de crescer como valor social. Desta forma, as políticas de diversidade e inclusão ganham destaque, seja para atrair novos talentos para empresas ou para contribuir no desenvolvimento de políticas de gestão de habilidades e competências a partir da diversidade.

A diversidade étnico-racial composta por pessoas negras (pretas e pardas) têm impulsionado no Brasil uma forte campanha de inclusão e representatividade em virtude da composição étnica da população brasileira e seu reflexo nos espaços empresariais e acadêmicos. Para além disso, o destaque que movimentos de tecnologistas negros vêm alcançando demostram que a diversidade étnico-racial, tal como a diversidade de gênero, é capaz de agregar valor aos times e organizações através das habilidades técnicas somadas à experiência que a vivência da negritude conduz.

As organizações de desenvolvimento de software estão dando passos importantes para mudar a maneira como constroem seus negócios, gerenciam suas equipes e desenvolvem seus produtos. Essas mudanças implicam em uma transformação no interior das organizações, que envolve a conscientização dos líderes, o reconhecimento das discriminações estruturais e o desenvolvimento de estratégias que busquem o desenvolvimento efetivo de um ambiente organizacional diverso e inclusivo.

Desta forma, as contribuições esperadas deste trabalho são: maior visibilidade das implicações da ausência ou presença da diversidade étnico-racial em times de desenvolvimento de software; o modelo de gestão de diversidade étnico-racial; criação de uma base de conhecimento sobre pesquisa-ação e gestão da diversidade em times de desenvolvimento de software; e, por fim, que este trabalho fortaleça as comunidades de diversidade étnico-racial da área da computação e as encoraje em suas pesquisas.

\section{Referências}

Andersson, C. and Runeson, P. (2007). A spiral process model for case studies on software quality monitoring-method and metrics. Software Process: Improvement and Practice, 12(2):125-140.

Aydemir, F. B. and Dalpiaz, F. (2018). A roadmap for ethics-aware software engineering. In 2018 IEEE/ACM International Workshop on Software Fairness (FairWare), pages 15-21. IEEE. 
Bradley, J. H. and Hebert, F. J. (1997). The effect of personality type on team performance. Journal of Management Development.

Chanlat, J.-F., Dameron, S., Dupuis, J.-P., de Freitas, M., and Ozbilgin, M. (2013). Management et diversité: lignes de tension et perspectives. Management international/International Management/Gestiòn Internacional, 17:5-13.

Coughlan, P. and Coghlan, D. (2002). Action research for operations management. International journal of operations \& production management.

Faraj, S. and Sproull, L. (2000). Coordinating expertise in software development teams. Management science, 46(12):1554-1568.

Fendler, J. and Winschiers-Theophilus, H. (2010). Towards contextualised software engineering education: an african perspective. In Proceedings of the 32nd ACM/IEEE International Conference on Software Engineering-Volume 1, pages 599-607.

Garrison, G., Wakefield, R. L., Xu, X., and Kim, S. H. (2010). Globally distributed teams: The effect of diversity on trust, cohesion and individual performance. ACM SIGMIS Database: the database for Advances in Information Systems, 41(3):27-48.

Gomes, N. L. et al. (2005). Alguns termos e conceitos presentes no debate sobre relações raciais no brasil: uma breve discussão. Educação anti-racista: caminhos abertos pela Lei Federal, 10639(03):39-62.

Hodgson, A., Hubbard, E.-M., and Siemieniuch, C. E. (2012). Toward an understanding of culture and the performance of teams in complex systems. IEEE Systems Journal, 7(4):606-615.

J. Highsmith, L. L. and Robinson, D. (2019). EDGE Value-Driven Digital Transformation. Addison-Wesley.

Kohl, K. and Prikladnicki, R. (2018). Perceptions on diversity in brazilian agile software development teams: a survey. In 2018 IEEE/ACM 1st International Workshop on Gender Equality in Software Engineering (GE), pages 37-40. IEEE.

Leonard, S. E., Pearcy, B. M., Shehab, R. L., and Walden, S. E. (2013). Minority student informed retention strategies. In 2013 IEEE Frontiers in Education Conference (FIE), pages 567-573.

Menezes, A. and Prikladnicki, R. (2018). Diversity in software engineering. In Proceedings of the 11th International Workshop on Cooperative and Human Aspects of Software Engineering, CHASE '18, page 45-48, New York, NY, USA. Association for Computing Machinery.

Page, S. E. (2019). The diversity bonus: How great teams pay off in the knowledge economy. Princeton University Press.

Staron, M. (2020). Action Research in Software Engineering. Springer.

Susman, G. I. and Evered, R. D. (1978). An assessment of the scientific merits of action research. Administrative science quarterly, pages 582-603.

Wohlin, C., Höst, M., and Henningsson, K. (2003). Empirical research methods in software engineering. In Empirical methods and studies in software engineering, pages 7-23. Springer. 